SPRINGER BRIEFS IN

ELECTRICAL AND COMPUTER ENGINEERING

Lei Lei

Chuang Lin

Zhangdui Zhong

Stochastic Petri

Nets for Wireless

Networks

Springer 


\section{SpringerBriefs in Electrical and Computer Engineering}



Lei Lei • Chuang Lin • Zhangdui Zhong

Stochastic Petri Nets

for Wireless Networks

Springer 
Lei Lei

State Key Laboratory of Rail

Traffic Control and Safety

Beijing Jiaotong University

Beijing, China

\section{Zhangdui Zhong \\ School of Computer and Information Technology \\ Beijing Jiaotong University \\ Beijing, China}

\author{
Chuang Lin \\ Department of Computer Science \\ and Technology \\ Tsinghua University \\ Beijing, China
}

$\begin{array}{lll}\text { ISSN 2191-8112 } & \text { ISSN 2191-8120 (electronic) } \\ \text { SpringerBriefs in Electrical and Computer Engineering } & \\ \text { ISBN 978-3-319-16882-1 } & \text { ISBN 978-3-319-16883-8 } & \text { (eBook) } \\ \text { DOI 10.1007/978-3-319-16883-8 } & & \end{array}$

Library of Congress Control Number: 2015937432

Springer Cham Heidelberg New York Dordrecht London

(C) The Author(s) 2015

This work is subject to copyright. All rights are reserved by the Publisher, whether the whole or part of the material is concerned, specifically the rights of translation, reprinting, reuse of illustrations, recitation, broadcasting, reproduction on microfilms or in any other physical way, and transmission or information storage and retrieval, electronic adaptation, computer software, or by similar or dissimilar methodology now known or hereafter developed.

The use of general descriptive names, registered names, trademarks, service marks, etc. in this publication does not imply, even in the absence of a specific statement, that such names are exempt from the relevant protective laws and regulations and therefore free for general use.

The publisher, the authors and the editors are safe to assume that the advice and information in this book are believed to be true and accurate at the date of publication. Neither the publisher nor the authors or the editors give a warranty, express or implied, with respect to the material contained herein or for any errors or omissions that may have been made.

Printed on acid-free paper

Springer International Publishing AG Switzerland is part of Springer Science+Business Media (www. springer.com) 
Recommended by Xuemin (Sherman) Shen 



\section{Preface}

Stochastic Petri Nets (SPNs), introduced in 1980, are a modeling formalism that can be conveniently used for the performance and reliability evaluation of discrete event systems. They admit a graphical representation that is well suited to top-down and bottom-up modeling of complex systems, and present a very straightforward mapping between events in the SPN model and events in the underlying Markov process. Although SPNs have become a useful tool for researchers in computer science, they are unknown to most wireless researchers and are not widely used to model wireless communication systems. On the other hand, the next-generation wireless networks such as the 5th Generation $(5 \mathrm{G})$ cellular systems will become increasingly complex in order to support for an increasingly diverse set of services, applications, and users - all with extremely diverging performance requirements. Since SPNs are found to be powerful in modeling performance of computer systems with a wealth of numerical solution techniques, it is very interesting to explore their applicability in wireless systems. This book was motivated by a desire to bridge the gap between the research on SPN modeling formalism and on the performance modeling of wireless networks.

In this book, we present our research results on applying SPNs to the performance evaluation of wireless networks under bursty traffic, in terms of typical Quality-of-Service (QoS) performance metrics such as mean throughput, average delay, packet dropping probability, etc. In the first chapter, we introduce the key motivations, challenges, and state-of-the-art research on using SPNs for cross-layer performance analysis in wireless networks. In Chap. 2, we first introduce the SPN basics, and then focus on two powerful techniques in SPNs to deal with the wellknown state space explosion problem: (1) model decomposition and iteration; (2) model aggregation using Stochastic High-Level Petri Nets (SHLPNs). We apply the first technique to the performance analysis of opportunistic scheduling and Device-to-Device (D2D) communications with full frequency reuse between D2D links in Chaps. 3 and 4, respectively. The above two scenarios show two typical radio resource sharing paradigms in wireless networks: orthogonal sharing by scheduling and non-orthogonal sharing by frequency reuse. We show that SPNs can provide an intuitive and efficient way in modeling the multiuser wireless system, 
especially facilitating the inclusion of different resource sharing paradigms between wireless links. Moreover, the original complex model whose state space grows exponentially with the number of users can be decomposed into multiple single user subsystems, and iteration methods can be used for performance approximation. In Chap. 5, we apply the second technique to formulate a wireless channel model for Orthogonal Frequency Division Multiplexing (OFDM) multi-carrier systems with SHLPN formalism in order to simplify the cross-layer performance analysis of modern wireless systems. Compared with existing Finite State Markov Channel (FSMC) model whose state space grows exponentially with the number of OFDM subchannels, our proposed SHLPN model uses state aggregation technique to deal with this problem. Closed-form expressions to calculate the transition probabilities among the compound markings of the SHLPN model are provided. When applied to derive the performance measures for OFDM system, the SHLPN model can accurately capture the correlated time-varying nature of wireless channels. We believe the example applications of SPNs to wireless networks and related findings will reveal useful insights for the design of radio resource management algorithms and spur a new line of thinking for the performance evaluation of future wireless networks.

Beijing, China

Lei Lei Chuang Lin Zhangdui Zhong 


\section{Acknowledgements}

The authors would like to acknowledge the support of the NSFC (Projects No. 61272168, No. U1334202, and No. 61472199), the State Key Laboratory of Rail Traffic Control and Safety in Beijing Jiaotong University (No. RCS2014ZT10), and the Key Grant Project of Chinese Ministry of Education (No. 313006). Thanks also are due to the Master candidates: Miss Qingyun Hao and Mr. Huijian Wang for their contribution of editing work.

A very special thanks to Prof. Xuemin (Sherman) Shen, the SpringerBriefs Series Editor on Wireless Communications. This book would not be possible without his kind support during the process. Thanks also to the Springer Editors and Staff, all of whom have been exceedingly helpful throughout the production of this book. 



\section{Contents}

1 Introduction ............................................. 1

1.1 Cross-Layer Performance Analysis of Wireless Networks

Using Stochastic Models ................................ 1

1.2 Motivations and Challenges on Using SPNs

for Performance Evaluation ................................ 3

1.3 Related Works on SPNs for Wireless Networks .................. 4

1.3.1 Ad Hoc Networks ................................. 4

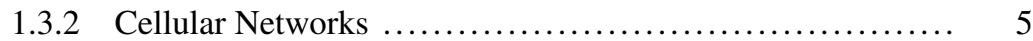

1.3.3 Multi-hop Wireless Networks ........................ 5

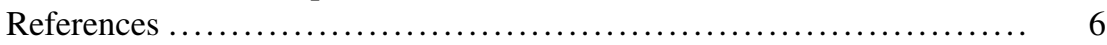

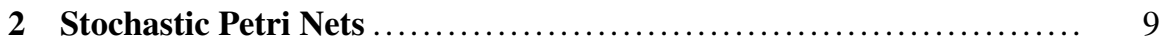

2.1 SPN Basics ............................................... 9

2.2 Model Decomposition and Iteration Technique ................. 12

2.3 Stochastic High-Level Petri Nets ............................. 15

References ............................................... 18

3 Performance Analysis of Opportunistic Schedulers Using SPNs ....... 19

3.1 Packet Level Performance Analysis of Opportunistic Schedulers ... . 19

3.2 The DSPN Model Formulation ............................ 21

3.2.1 The M/MMDP/1/K Queuing System .................. 21

3.2.2 The DSPN Model ................................. 22

3.2.3 Scheduling Strategies ............................... 23

3.3 Model Solution and Performance Analysis .................... 25

3.3.1 Single User System ............................... 25

3.3.2 Model Decomposition and Iteration .................. 27

3.4 Numerical Results..................................... 33

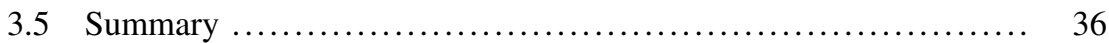

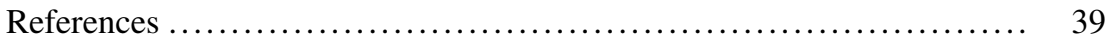


4 Performance Analysis of Device-to-Device Communications with Dynamic Interference Using SPNs ....................... 41

4.1 Packet Level Performance Analysis of D2D Communications ....... 41

4.2 The Coupled Processor Queueing System ..................... 44

4.3 Steady-State Solution of the Queueing System.................. 48

4.3.1 Transition Probability of the Queue State ............... 50

4.3.2 Transition Probability of the Server State ................ 50

4.3.3 Steady-State Probability of Markov Chain ............... 58

4.4 Model Decomposition and Performance Approximation

Using DSPNs ............................................ 58

4.4.1 The DSPN Model ................................... 58

4.4.2 Model Decomposition and Iteration ..................... 60

4.5 Numerical Results....................................... 62

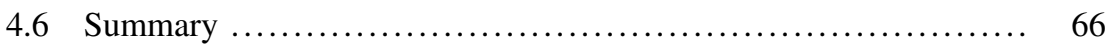

References ................................................. 76

\section{Packet Level Wireless Channel Model}

for OFDM System Using SHLPNs ............................... 79

5.1 Packet Level Wireless Channel Model ........................... 79

5.2 SHLPN Model of OFDM Wireless Channel..................... 81

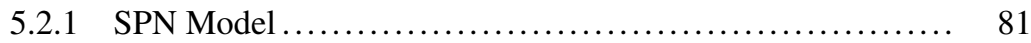

5.2.2 SHLPN Model .................................... 83

5.3 Example Application to Cross-Layer Performance

Analysis of Cellular Downlink ................................ 89

5.3.1 Model Description ................................. 89

5.3.2 Numerical Results ................................... 91

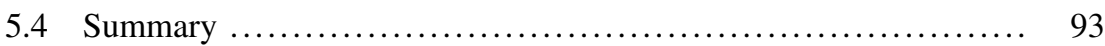

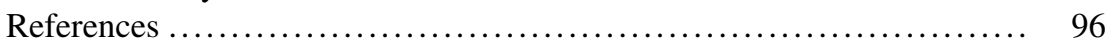

6 Conclusions and Outlook ...................................... 99

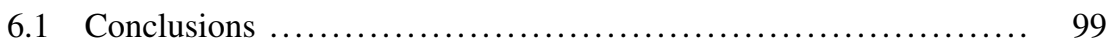

6.2 Outlook ................................................. 100

References .............................................. 101 


\section{Acronym}

\begin{tabular}{|c|c|}
\hline $3 \mathrm{G}$ & 3rd-Generation \\
\hline 3GPP & 3rd Generation Partnership Project \\
\hline $4 \mathrm{G}$ & 4th Generation \\
\hline $5 \mathrm{G}$ & 5th Generation \\
\hline AMC & Adaptive Modulation and Coding \\
\hline $\mathrm{BS}$ & Base Station \\
\hline $\mathrm{CA}$ & Channel-Aware \\
\hline CAC & Call Admission Control \\
\hline CDMA & Code Division Multiple Access \\
\hline CPN & Colored Petri Net \\
\hline CQA & Channel/Queue-Aware \\
\hline $\mathrm{CR}$ & Cognitive Radio \\
\hline СТMC & Continuous-Time Markov Chain \\
\hline D2D & Device-to-Device \\
\hline DCA & Dynamic Channel Allocation \\
\hline $\mathrm{DCF}$ & Distributed Coordination Function \\
\hline DEDS & Discrete Event Dynamic Systems \\
\hline DSPN & Deterministic and Stochastic Petri Net \\
\hline DTMC & Discrete-Time Markov Chain \\
\hline DTSPN & Discrete Time Stochastic Petri Net \\
\hline FSMC & Finite State Markov Channel \\
\hline FR & Full Reuse \\
\hline GE & Gilbert-Elliot \\
\hline GSPN & Generalized Stochastic Petri Net \\
\hline HLPN & High-Level Petri Net \\
\hline HSPDA & High Speed Downlink Packet Access \\
\hline ISI & Inter-Symbol Interference \\
\hline LCR & Level-Crossing Rate \\
\hline LTE & Long Term Evolution \\
\hline M2M & Machine-to-Machine \\
\hline MAC & Medium Access Control \\
\hline
\end{tabular}




$\begin{array}{ll}\text { MC } & \text { Markov Chain } \\ \text { MDP } & \text { Markov Decision Process } \\ \text { MDPN } & \text { Markov Decision Petri Net } \\ \text { MMDP } & \text { Markov Modulated Deterministic Process } \\ \text { MIMO } & \text { Multiple-Input Multiple-Output } \\ \text { M-QAM } & \text { M-ary Quadrature Amplitude Modulation } \\ \text { NRT } & \text { Non-Realtime } \\ \text { OFDM } & \text { Orthogonal Frequency Division Multiplexing } \\ \text { OFDMA } & \text { Orthogonal Frequency Division Multiple Access } \\ \text { OS } & \text { Opportunistic Scheduling } \\ \text { PF } & \text { Proportional Fair } \\ \text { PN } & \text { Petri Net } \\ \text { QA } & \text { Queue-Aware } \\ \text { QoS } & \text { Quality-of-Service } \\ \text { RR } & \text { Round-Robin } \\ \text { RT } & \text { Realtime } \\ \text { SHLPN } & \text { Stochastic High-Level Petri Net } \\ \text { SINR } & \text { Signal to Interference and Noise Ratio } \\ \text { SNR } & \text { Signal to Noise Ratio } \\ \text { SPN } & \text { Stochastic Petri Net } \\ \text { SRN } & \text { Stochastic Reward Net } \\ \text { SWN } & \text { Stochastic Well-Formed Petri Net } \\ \text { TDMA } & \text { Time Division Multiple Access } \\ \text { UE } & \text { User Equipment } \\ \text { UMTS } & \text { Universal Mobile Telecommunications System } \\ & \end{array}$

